\title{
MOBILE ROBOT MOVEMENT PLANNING AND CONTROL INTELLIGENT SYSTEM IN UNKNOWN ENVIRONMENT
}

\author{
Andrius Nemeiksis, Vitalijs Osadcuks \\ Latvia University of Life Sciences and Technologies, Latvia \\ nemeiksis.andrius@1lu.lv,.vtl@tvnet.lv
}

\begin{abstract}
Robot engineering and related technologies have been rapidly developing during the last decades providing more and more opportunities for using robots in various fields of human activity. In the first place, this is due to continuous improvement of the characteristics of robot engines, energy resources, on-board system calculation tools, and, most importantly, development of sensory tools. This not only improves the management of the robot movement, but also allows for developing custom control systems of advanced levels. At the beginning of this century, the most rapid development in the field of mobile robots was observed in autonomous mobile robots that function in an unknown environment, which constitute the research object of this article.

Therefore, the purpose of this article is to prepare the intelligent systems development methods for mobile robot motion planning, which ensure that the robot performs a planned and adjusted during the movement safe trajectory in an environment with unfamiliar obstacles. In order to achieve this purpose, a theoretical research of the mechatronic mobile robot's movement in an unknown environment was carried out, based on which the corresponding results of the research have been obtained, i.e. the theoretical operative control systems for mobile robot's movement in an unknown environment have been tested in real practical situation by designing a theoretical scheme for this research that consists of a laboratory model of a bi-cycle mobile robot and a special algorithm for its operative control. The results of the research evaluate the effectiveness of such mobile robot operation, also they show the progress and resutls of the theoretical experiment in unknown environment with certain obstacles set out in random places on the mobile robot path, as well as they identify the main factors how to improve the experimental research base.
\end{abstract}

Keywords: localization tasks, odometric or visible information, point-to-point or continuous path motion, stationary and dynamic environment.

\section{Introduction}

The tasks of a mobile robot following its path include: motion control, path planning, mapping and localization. Autonomy is assigned to the main tasks, related to the mobile robot use, the solution of which is relevant worldwide. The main problems are the following: mobile robot motion in a dynamic environment, localization quality improvement, mapping, etc.

Robot motion is usually executed in accordance with a predetermined path, which is indicated by the line on the floor or by other directives. There are two existing techniques of the mobile robot motion control [1;2]:

- "point-to-point" motion (PTP), when the initial and final motion points are indicated, and the path is marked by directives or limiting devices;

- continuous path motion (CP), when the mobile robot coordinates are being tracked in realtime and adjusted if necessary.

The first control technique (PTP) is usually used, when there is no need to estimate the actual position and direction of a robot following its path. However, when the robot moves, the changes in the surrounding working environment, which can cause an impact or accident, shall be considered. The positive factor, while applying PTP motion control, is the relative simplicity and speed of the respective calculations, which are related to the fact that there is no need to solve an inverse kinematics task [2; 3]. Respectively, CP control technique is more susceptible to resources than PTP and requires a more powerful calculation device, because each iteration step has an inverse kinematics task to solve for further mobile robot motion. CP is used to perform tasks more accurately $[1 ; 2 ; 4]$.

While moving along the path, the mobile robot moves from the initial position to the final one. Mobile robot path is a geometric locus of points, which is indicated by the movement of the point (in this case - the middle between the two rear wheels) as a time function in the Cartesian coordinate system [5].

The objective of the mobile robot path planning is to transform the technical conditions of a specific task into the desirable robot path, when a mobile robot moves along a predetermined path in 
accordance with the controlling actions. Figure 1 presents the mobile robot path planning process algorithm [6].

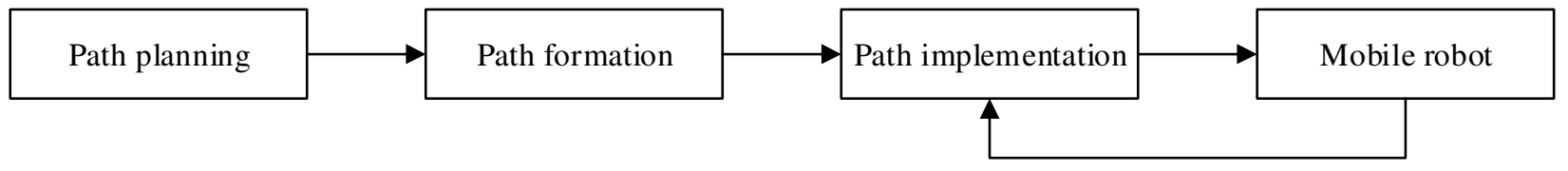

Fig. 1. Mobile robot path planning process algorithm

Mobile robot path can be indicated as a continuous time function or a discrete point sequence. Usually the path is determined in the Cartesian coordinate system, and in order to make it work the inverse kinematics equations are employed.

Path planning under the obstacle-free conditions or in a known environment is sometimes a tricky but rather easily solved task, primarily related to path planning. However, recently there has been a growing interest in autonomous mobile robots (AMR). First of all, it is related to the reconsideration of the control concept, when one of the most important factors becomes the human resource preservation and the necessity to perform various works in the extreme situation zones, where the presence of a human being is undesirable or prohibited. A foreign research analysis shows that:

- various size air, land and underwater deployment autonomous robots are being actively developed;

- in recent years, works related to mini and micro robotics projects have been initiated;

- intelligent technologies are being employed more actively, while developing autonomous objects, and the term "intelligent robots" is being used more often.

\section{Materials and methods}

The methods based on the classical theory of artificial intelligence are introduced in order to solve the localization tasks in various fields. The intelligent systems in such localization processes are considered to be the mobile robots of various types functioning in an unknown environment. This is related to the fact that the most significant development in the field of automatic solution methods of localization tasks concentrated on the elements of artificial intelligence. In order to navigate mobile robots operating in different environment, for instance, underwater or up in the air, the modified versions of the overground environment methods are applied [7-10].

The first method $[11 ; 12]$ - mapping of the environment is done by applying the environment mapping method, dividing the functional mobile robot outdoor environment to the set $\mathrm{P}$ of rectangular areas of various size, which either can move freely or have obstacles in them. In order to detect the obstacles, a mobile robot is equipped with the rangefinders. The coordinates of the areas are determined in accordance with the odometric subsystem data, based on the information about the wheels of a moving robot.

Originally, the outdoor environment is unexplored, and the robot starts investigating it by moving straight along the freely chosen direction. Upon detecting a new obstacle, the contour determination procedure $\mathrm{P}$ is performed in order to map it and divide it. To perform such task, the robot tracks the obstacle edges, by moving along its contour at a certain previously set distance, and marks the obstacle edge coordinates in the map.

When the obstacle contour is determined, its approximation in rectangles is performed. In addition, the space around the obstacle is also divided into rectangular areas. The vertex point coordinates of these areas are marked in the map. An example of outdoor environment division into rectangular areas is presented in Figure 2.

Upon determining the coordinates of the homogenous areas, the database $\mathrm{D}$ is created, where similar records, providing the information about the ability to move from one section to another: $\{\mathrm{A}$, B, flag\}, are stored. Here, A and B are the numbers of neighbouring sections, "flag" - a mark, which means "true", if it is possible to get from A to B, and "false" otherwise. For instance, the record of sections $\varepsilon$ and $\eta$ is the following: $\{\varepsilon, \eta$, false $\}$, and the record of sections $\alpha$ and $\beta-\{\alpha, \beta$, true $\}$. The localization graph, the vertices of which consist of sections, where a robot can move freely, is 
concluded in accordance to the data from D. An example of the aforementioned environment graph is presented in Figure 3.

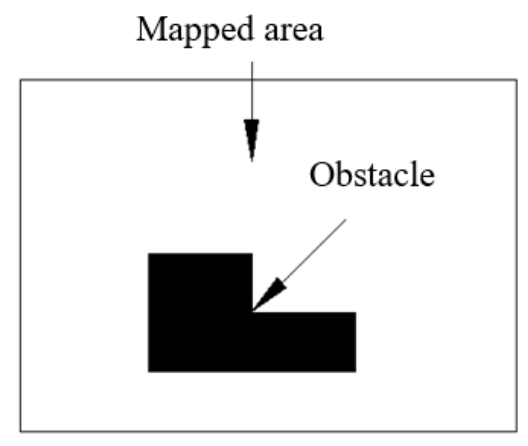

a

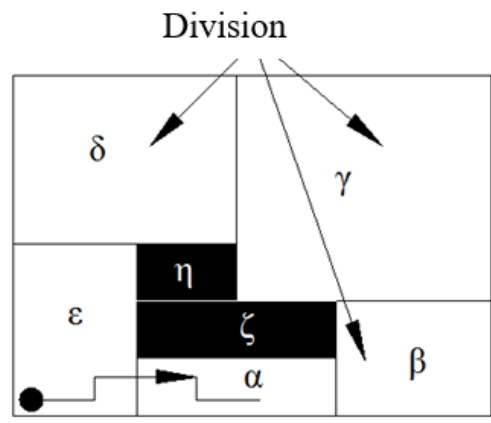

$\mathrm{b}$

Fig. 2. Example of outdoor environment division into rectangular areas

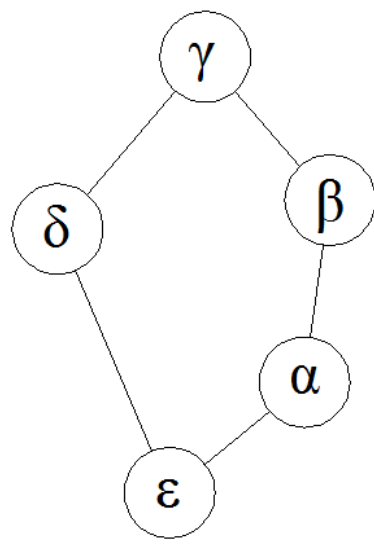

Fig. 3. Localization graph example

To plan the motion towards the target section the Dijkstra's algorithm, which finds the shortest path in the graph, is used. The path is planned in the following way. Let us say that a robot needs to go from section $\varepsilon$ to section $\alpha$, as indicated in Figure 3, b. The robot moves straight, along the horizontal wall $\varepsilon$, until it reaches the vertical wall $\alpha$. Then the robot moves along this wall and, upon crossing it in the middle point, moves forward, until it reaches the horizontal wall $\alpha$ and so on, as it is presented in Figure 3, b.

As it is seen from the method description, it is suitable to use it only in the stationary environment, where the obstacle edges are parallel or perpendicular to each other. The one-time selection of the shortest path from the initial area to the target area does not allow to plan the possibility of moving obstacles, and the motion towards the target, while tracking the walls in a certain area, does not guarantee the optimal path even in the stationary environment. The advantage of this method is that its implementation does not require extensive calculation.

The second method [13-16] - mapping of the environment is done by applying the histogram grid method. The point of this method is that the environment, in which the robot moves along the randomly selected path with the help of the acoustic rangefinders, is divided into square elements, referred to as squares. The environment map is a two-dimensional array of squares, aka histogram grid. Each square holds a certainty function value that it contains an obstacle. Let us say that a robot in square $C_{0}$ moves along the arrow direction, as it is presented in Figure 4, a.

Then the certainty function value is updated for each square that is in the acoustic rangefinder axis, that way: the distance $d_{\kappa}$ from the robot frame to the nearest obstacle is calculated. Later, based on the $d_{\kappa}$ value and the square size, it is calculated which square in the grid corresponds with the obstacle position. The certainty value of the square increases at a certain size $\Delta o$ (in this case $\Delta o=+3)$. The certainty value in other squares, located between the detected obstacle and CO, 
decreases by 1, as presented in Figure 4, a. When the robot repeats the path, the values in the histogram squares are modified once again and so on. The squares with the positive certainty function value depict the obstacle position, as presented in Figure 4, b.

Obstacle

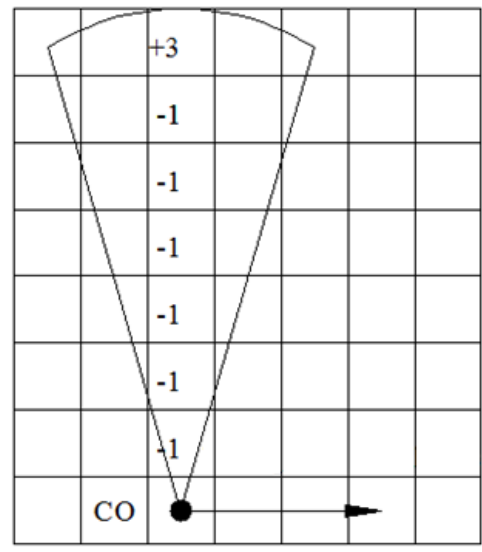

Histogram grid

a
Environment maps fragment

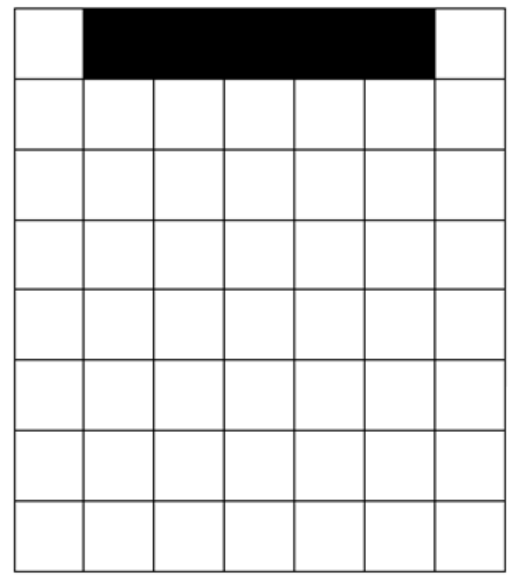

b

Fig. 4. Environmental mapping by applying histogram grid method

Upon investigating the environment, the elements of the two-dimensional histogram grid array, holding the positive value, depict the obstacle position, and the elements, holding the negative or zero value, represent the obstacle free space.

During the localization process, the motion direction towards the target is determined in accordance with the information in the map. In every step of the path planning in the environment map, the $W_{s} \times W_{s}$ square window is marked around the current robot position, which is determined by the odometric subsystem, as presented in Figure 5.

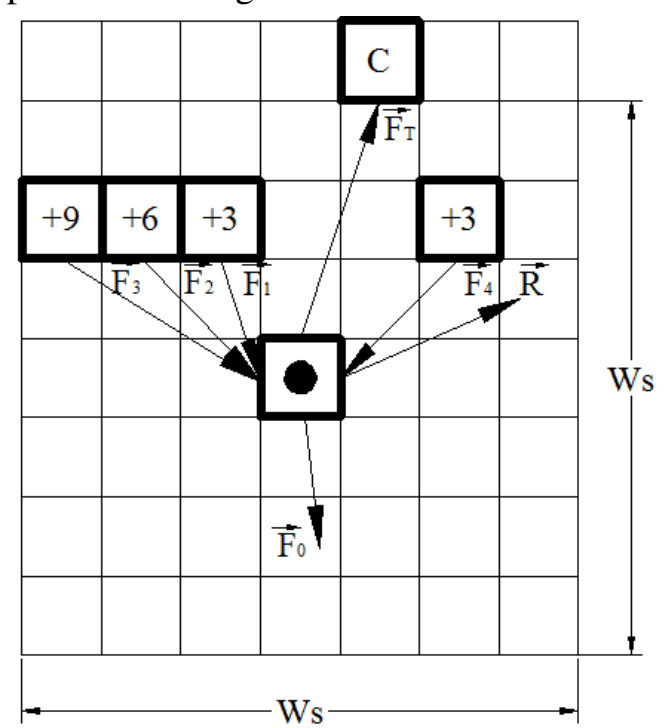

Fig. 5. Determining movement direction in accordance with environmental map

All the map areas, containing the information about the obstacles and entering the square, are called active. Every active area towards the current robot position has a repulsive force $\vec{F}_{i}$. The value of its module is calculated on the basis of the following equation:

$$
\left|\vec{F}_{i}\right|=\frac{C_{i j}}{d_{k}^{2}},
$$


where $C_{i j}$ - certainty function value, indicating that there is an obstacle in a specific square;

$d_{k}$ - distance from the square with an obstacle from the square with the current robot position in the map.

Later, the resultant of all the vectors $\vec{F}_{i}-\vec{F}_{0}$ is indicated (the figure shows that the square with the current robot position is influenced by the repulsive force $\vec{F}_{1}-\vec{F}_{4}$ ). Along with the repulsive forces, the same square is influenced by the constant force towards the target $-\vec{F}_{T}$. The direction towards the target is determined as the resultant of the vectors $\vec{F}_{0}$ and $\vec{F}_{T}$ (vector $\vec{R}$ in Figure 1.17.).

The motion planning towards the target, based only on the obstacle position data in the map, limits the method application to the static environments. In addition, the calculations increase together with the number of obstacles in a specific square. However, not only the static environment conditions can guarantee that the optimal path towards the target will be estimated, as the vector $\vec{R}$ direction does not necessarily correspond with the optimal motion direction towards the target, as in Figure 5. The positive side of this method is that the environment mapping process is relatively easy.

The third method [17] - the localization task solution method applied in residential and household premises. It works in the following way: the outdoor environment is analysed while moving along the obstacle edges, and the environmental features (indicators) are identified and recorded to the map. The following indicators are used: "wall on the right", "wall on the left", and "corridor".

The features are found based on the rangefinder and stopwatch data, and their interposition is determined by using an electronic compass. Moreover, the indicator "wall on the right" is identified, if during the set interval $\Delta T$ the rangefinder detects an obstacle on the right. The indicator "wall on the left" is identified, if during the set interval $\Delta T$ the rangefinder detects an obstacle on the left. Correspondingly, the indicator "corridor" is identified, if obstacles are detected on the left and on the right of the robot frame. The types of indicators are presented in Figure 6.

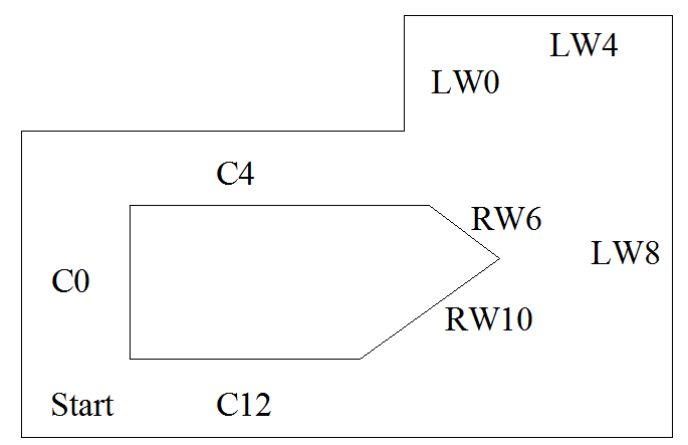

Fig. 6. Outdoor environment indicators used in mapping

In this figure, $\mathrm{C} 0, \mathrm{C} 4$, and $\mathrm{C} 12$ indicate a corridor, LW0, LW4, and LW8 indicate the walls on the left, RW6, RW10 - the walls on the right, Start is the initial position of the robot before it starts investigating the environment. The indicators are detected during the environment analysis. Every time the indicator is found, its descriptor is created, and it holds the following information: indicator type (T) "wall on the right", "wall on the left", and "corridor"); motion direction $(\Theta)$, which leads to the further indicator, when you are at the previous one; indicator "length" $(L)$; approximate estimation of the robot position in accordance with the indicator $P(x, y)$.

Later, the descriptor data, corresponding with the new indicator, are compared to the descriptor data that are in the map. If the data stored in the new descriptor significantly differ from the data stored in the map, the map is updated by adding a new descriptor. The environmental map is an undirected graph, which vertices correspond with the descriptors, and the nodes mean that the indicators are near. Figure 7 presents the map of the environment depicted in Figure 6. 


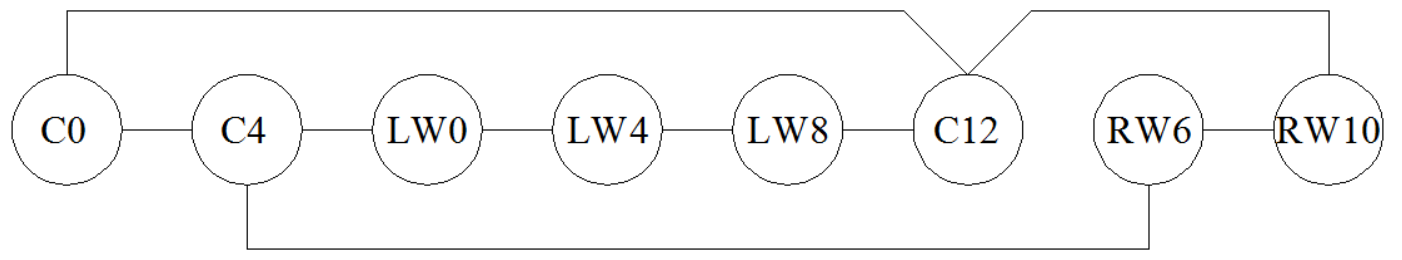

Fig. 7. Outdoor environment map

During the localization process, the actual position in the environmental map is determined by comparing the information regarding the distance to the obstacle with the information stored in the descriptors. The current robot position is considered to be the area, which information is the most similar to the current sensor information. The path towards the target is planned by applying the wave diffraction method. Simultaneously, the adjacency matrix is created based on the information about the adjacency of the map nodes. An example of the adjacency matrix is presented in Figure 8. In this figure, the elements representing the adjacent vertices are depicted in white and the elements representing the non-adjacent vertices are depicted in black.

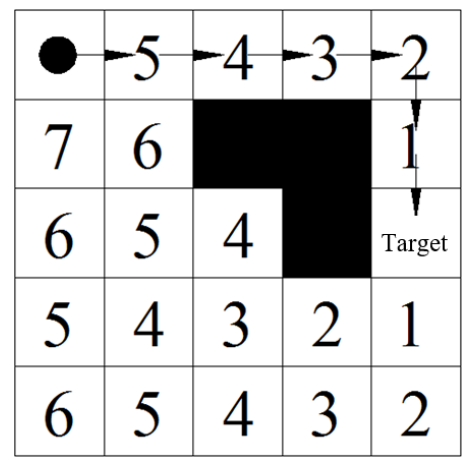

\section{Fig. 8. Illustration of wave diffraction process}

In accordance with the four-point neighbourhood model, the stimulating wave is sent from the target area in multiple directions in order to reach the robot area. Every matrix element stores the diffraction process number, as it is presented in Figure 8. After the diffraction process, the matrix element having the smallest number is selected in accordance with the four-point neighbourhood model, and the robot moves towards the direction of the vertex representing that element. Upon reaching the vertex, the process is repeated until the target area is reached. An example of the sequence is marked with arrows in Figure 8.

The described method is intended for use in the environments, where the obstacle and target positions are known beforehand. It could be difficult for a robot to determine its own position in the environmental map, if there were any mobile obstacles in its path. The advantage of this method is that its implementation does not require extensive calculation.

The main idea of the fifth mapping method is that the map graph vertices have to provide information about the neighbouring areas in the environment in order to reduce the error while estimating the actual position. For this purpose, they have to depict the interposition of the areas in the outdoor environment. In addition, each vertex stores the information from the visible subsystem, for instance, digital photos of the space surrounding the area that corresponds with the vertex in the map. Having this data, the robot determines its own position by comparing the current visible information with the information that is stored in the map graph vertex. The vertex of the current robot position is the one, which best corresponds with the data. In order to form the environmental map, the robot periodically moves along the freely chosen direction at the distance equal to its frame length, while including new vertices in the map and saving the visible information. Then, it is determined which vertices are neighbouring to the newly created one. Each neighbouring vertex is connected to the newly created using joints, which store the information about the needed distance and direction in order to move from one vertex in the map to another. An example of an outdoor environment and its map, created using the aforementioned method, are presented in Figures 9, a-b. 


\section{Topological map}

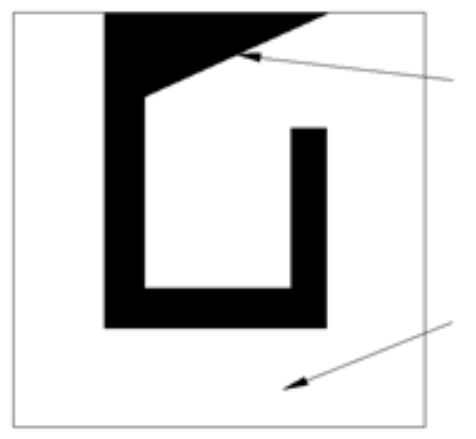

a

\section{Obstacle \\ Mapped area}

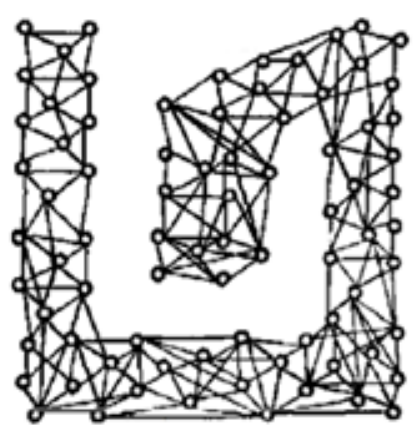

b

Fig. 9. Outdoor environment and its topological map

Following the foregoing approach, the robot determines its own position by calculating the probability of being in each of the nodes of the map, which are estimated comparing the current sensor data to the data included in the map. Upon calculating the probability values for each node in the map, they are compared to the threshold that was set beforehand. Finally, the set of nodes, having higher than the threshold probability, are analysed as the most probable robot position, as it is presented in Figure 10, where the white balls represent the vertices of the probable robot position.

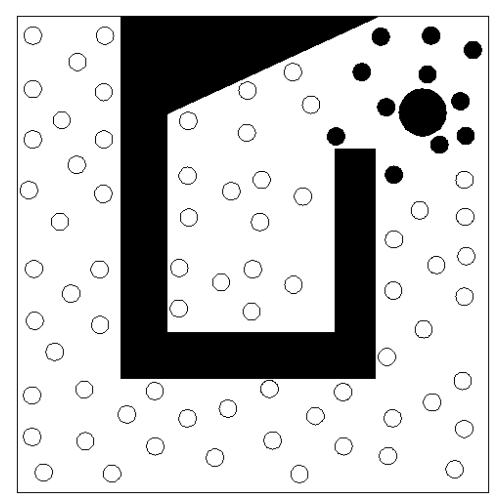

Fig. 10. Determining agent position in environmental map

Later, the node, having the maximum probability value, is selected from the set and is considered to be the current robot position. Upon determining the actual position, the path from the current map node to the target node is planned in accordance with the modified Dijkstra's algorithm.

The main drawbacks of the described method are the extensive calculations while determining the robot position and forming the environmental map, due to which it is difficult to plan the path in realtime, as well as disregarding environment dynamics while planning the path towards the target.

The sixth mapping method [18-20] tries to create the localization subsystem for a robot in a dynamic environment. The principle of this method is that the robot, while moving in the environment, detects its dividing points, referred to as indicators, and includes their dividing settings in the environmental map, which takes the form of a graph. In addition, the graph node stores the data regarding its coordinates as well as the movement sequence and duration, which is needed for a robot to reach the next indicator.

The outdoor environment investigation method can be described in the following sequence [18]:

1. Indicators are located in the blind spots of the environment, at the corridor intersections, as well as near the doors;

2. If two motion directions exist (forward - backward), the chosen direction is the one corresponding to the current direction; 
3. If more than two motion directions exist and there is no indicator in the current robot position, the planning system creates a new indicator and sets the connection with the previous one (saves the data regarding the motion direction towards the new indicator and the length of the path in order to reach the indicator);

4. If the path intersection in the current robot position has an indicator, the neuro-network plans the optimal path towards the indicator, which is the closest to the target;

5. If there is an indicator "path intersection" in the current robot position and at least one unexplored motion direction, the localization subsystem will try to investigate whether it is a shorter path towards the target;

6. If there is no clear path between two indicators, the connection between them is eliminated.

In accordance with the foregoing method, the robot analyses the environment and forms the map until it reaches the target. The described path is used until the obstacle is detected. This case is presented in Figures 11, a-b, where the environment is a single building floor. The path towards the target, estimated during the initial environment analysis, is presented in Figure 11, a. Upon repeating the path the second time, the robot faced an obstacle - a closed door. Facing the obstacle, the robot updates the environment analysis in order to find a clear path and avoid the obstacle, as depicted in Figure 11, b.

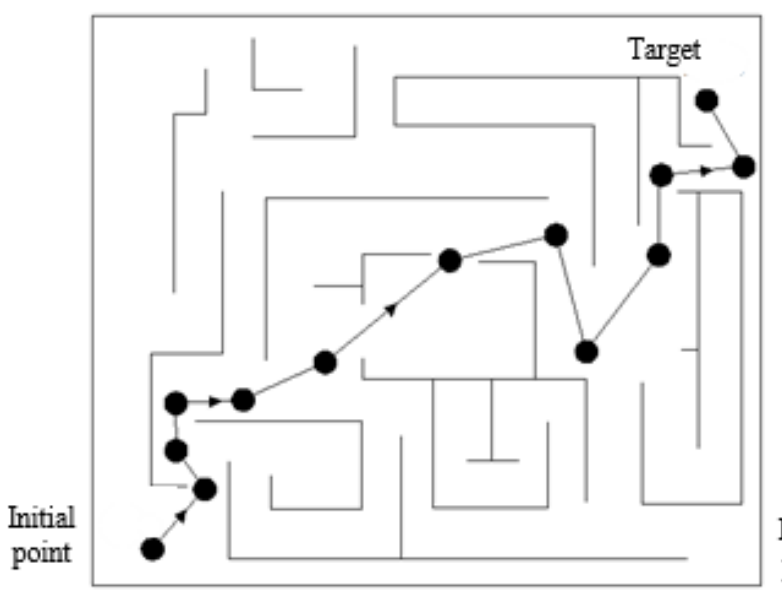

a

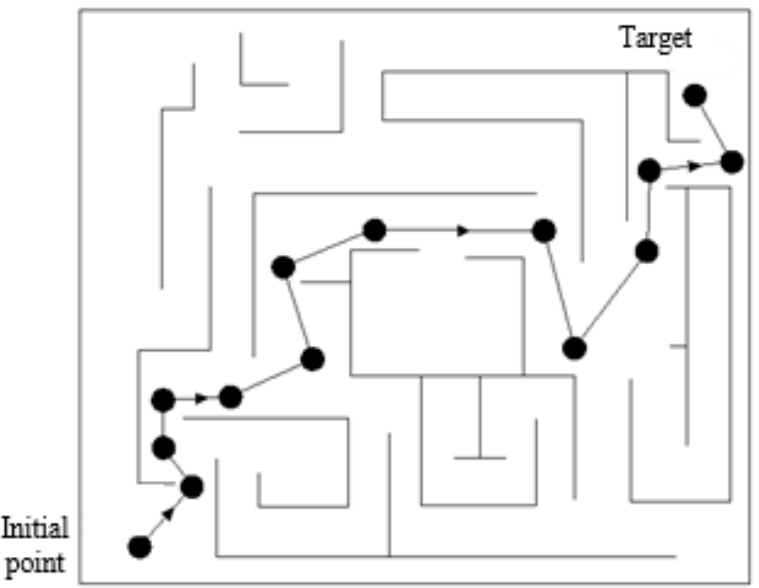

b

Fig. 11. Example of localization system operation

The drawback of the aforementioned method is that the method can only be used in the quasistationary outdoor environments that have been formalized beforehand.

\section{Discussion}

The intellectual mobile systems are developed in science centers of Japan, the USA, the UK, France, Germany and other countries. However, despite all these efforts, the results are far from what they are expected. This is due to the fact that in the general cases the provision of automated navigation of the intelligent mobile system involves the necessity to create algorithms that allow solving the problems related to formation of the permeability map of the pre-informal environment; identifying the current position on the map based on the information from the environment; planning the movement towards the target trajectory using the map.

In turn, the solution of these problems is of fundamental scientific-practical nature $[7 ; 8]$ : adequate environmental perception; precise presentation of knowledge about it; effective implementation of precise actions of efector subsystem in real-time.

So, as seen in the described method analysis, they allow to solve localization tasks only under the stationary environment conditions. Meanwhile, the majority of methods require prior information about the interposition of the objects in the environment. A lot of the mentioned methods do not allow to plan the optimal path even under the stationary environment conditions, and none of these methods analyse the target behaviour. Only the odometric or visible information is used to determine the actual 
robot position, which causes great errors that are difficult to predict. While using the aforementioned methods to solve the localization tasks in the non-stationary environment, we face the major difficulties related to the fact that none of them allows a robot to predict its path in order to avoid the collision with mobile obstacles.

In addition, while trying to create methods that have no mentioned drawbacks and are properly realised by traditional architectures, based on the coherent information processing, we face the fact that it is impossible to ensure the robot functioning in real-time. This problem can be solved by creating localization subsystems based on the neuro-network information processing methods.

\section{Conclusions}

To summarise the analysis of the robot motion planning in an unknown environment, discussed in this chapter, the following conclusions can be drawn:

1. The application of a certain motion planning method depends on the robot motion velocity. If the robot moves quickly or the motion is planned in a dynamic environment, the real-time mode is to be applied, because under the unpredictable robot environment conditions the decisions have to be made quickly.

2. While processing the information from the sensors, the environmental model update is implemented step by step or during every program iteration. The speed of this process determines the ability to efficiently solve the problem of the robot motion planning in the environment with unknown mobile obstacles.

3. There is a necessity to apply the artificial intelligence methods in the decision making stage, during the estimation of the robot's path points in the operational robot area in order to move from the initial configuration to the target configuration while avoiding obstacles.

4. While planning the robot motion in an unknown environment in the real-time mode, especially with unknown mobile obstacles, the time consumption is very important. It includes the time intended for the respective calculations, the time, during which the sensors scan the information about the environment, and the time intended to process this information, which depends directly on the type of the range sensor. So, choosing the right sensors is the most important part of the mobile robot motion planning process in an unknown environment and its realization in practice.

\section{References}

[1] Hartanto R. A hybrid deliberative layer for robotic agents: fusing DL reasoning with HTN planning in autonomous robots. Springer-Verlag. Berlin: Heidelberg, 2011. 215 p.

[2] González R, Rodriguez, F., Guzmán, J.L. Autonomous tracked robots in planar off-road conditions. Modelling, Localization, and Motion Control. Springer-Verlag. Berlin: Heidelberg, 2014. 119 p.

[3] González R., Fiacchini M., Guzmán J.L., Alamo T., Rodrıguez F. Robust Tube-based Predictive Control for Mobile Robots in Off-Road Conditions. Robotics and Autonomous Systems vol. 59, no. 10, 2011, pp. 711-726.

[4] Nikitenko A., Liekna A., Andersone I., Ekmanis M., Urtans E. Mobile robot path planning for indoor use. Proceedings of $14 \mathrm{~h}$ International scientific conference „Engineering for rural development", May 29-30, 2014, Jelgava, Latvia, pp. 366-372.

[5] Siciliano B. Handbook of Robotics. Springer-Verlag. Berlin: Heidelberg, 2008. 1611 p.

[6] Tahirovic A., Magnani G. Passivity-based model predictive control for mobile vehicle motion planning. Proceedings of IEEE/RSJ International Conference on Intelligent Robots and Systems, October 18-22, 2010, Taipei, Taiwan, pp. 307-312.

[7] Zhang H., Shi Z., Wei X. A rapid path planning adaptive optimization algorithm based on fuzzyneural network for multi-robot systems. Proceedings of "International conference on cyberspace technology (CCT 2013)", November 22-23, 2013, Beijing, China, pp. 32-38.

[8] Tingbin C., Qisong Z. Robot motion planning based on improved artificial potential field. Proceedings of 2013 3rd International Conference on Computer Science and Network Technology, October 12-13, 2013, Dalian, China, pp. 1208-1211. 
[9] Nagata F., Otsuka A., Watanabe K., Habib M.K. Multiple mobile robots system with networkbased subsumption architecture. International Journal of Mechatronics and Manufacturing Systems, vol. 6, no. 1, 2013, pp. 57-71.

[10] Li T., Yuan G., Duan Q. Navigation technology of autonomous mobile robots in unknown environments. Proceedings of 2010 IEEE/ASME International Conference on Mechatronic and Embedded Systems and Applications, July 15-17, 2010, Qingdao, ShanDong, China, pp. 533-538.

[11]Li Z., Deng J., Lu R., Xu Y., Bai J., Su C.Y. Trajectory-tracking control of mobile robot systems incorporating neural-dynamic optimized model predictive approach. IEEE Transactions on Systems, Man, and Cybernetics: Systems, vol. 46, no. 6, 2016, pp. 740-749.

[12] Hong-Ming W., Zeng-Guang H., Jia M., Yun-Chu Z., Yong-Qian Z., Min T. Sonar feature map building for a mobile robot. Proceedings of 2007 IEEE International Conference on Robotics and Automation. April 10-14, 2007, Roma, Italy, pp. 4152-4157.

[13] Wai R.J., Lin Y.W. Adaptive Moving-Target Tracking Control of a VisionBased Mobile Robotvia a Dynamic Petri Recurrent Fuzzy Neural Network. IEEE Transactions on Fuzzy Systems, vol. 21, no. 4, 2013, pp. 688-701.

[14]Ferrier J.L., Bernard A., Gusikhin O., Madani K. Informatics in Control, Automation and Robotics. Springer-Verlag. Berlin: Heidelberg, 2013. 228 p.

[15] Kuderer M., Kretzschmar H., Burgard W. Teaching mobile robots to cooperatively navigate in populated environments. Proceedings of IEEE/RSJ International Conference on Intelligent Robots and Systems (IROS), November 3-7, 2013, Tokyo, Japan, pp. 3138-3143.

[16] Kaneshige A., Nagai S., Ueki S., Miyoshi T., Terashima K. An algorithm for online path planning of autonomous mobile overhead traveling crane based on obstacle information. Proceedings of 2012 IEEE International Conference on Automation Science and Engineering (CASE), August 20-24, 2012, Seoul, South Korea, pp. 914-919.

[17] U. Ruckert, J. Sitte, F. Werner. Advances in autonomous mini robots. Springer-Verlag. Berlin: Heidelberg, 2012. 280 p.

[18] Gao Q., Cho Y.I. A Dynamic ontology-based multi-agent context-awareness user profile construction method for personalized information retrieval. International journal of Fuzzy logic and intelligent systems, vol. 12, no. 4, 2012, pp. 270-276.

[19]Zhang H., Shi Z., Wei X. A rapid path planning adaptive optimization algorithm based on fuzzyneural network for multi-robot systems. Proceedings of "International conference on cyberspace technology (CCT 2013)", November 22-23, 2013, Beijing, China, pp. 32-38.

[20] Boonphoapichart, S., Komada, S., Hod, T. Robot's motion decision-making system in unknown environment and its application to a mobile robot. Proceedings of 2002 IEEE International Conference "Industrial Technology", December 11-14, 2002, Tsu, Japan, pp. 18-23. 